falls mit einer App: „ProSecure ${ }^{\varpi}$ Pro“. Mehr Geräte sind nicht notwendig, der Tablet-PC oder das Smartphone fungieren nämlich gleichzeitig als Batterie.

CardioSecur ${ }^{\circledast}$ hat ein CE-Siegel und ist ein anerkanntes Medizinprodukt der Klasse IIa. Die FDA-Zulassung stehe ante portas, hieß es auf der Medica. Geld verdient das Unternehmen mit dem Verkauf des Features. Patienten zahlen zum Beispiel 9,95€ pro Monat.

iFeel Labs will mit seiner App „iFeel Labs Match 3“ sogar die Therapie in die mobile Welt verschieben. Über ein Smartphone-Spiel und einen Biofeedback-Sensor sollen Asthmapatienten in Stresssituationen ruhiges Atmen lernen. Die Lösung soll den Inhalator ersetzen und ist FDA sowie CE-geprüft.

Komplett ersetzt wird der Arzt durch die neue Technik jedoch nicht, das machten Patienten-, Pharma- und Medizinvertreter auf einer Podiumsdiskussion beim Medica Health IT Forum deutlich. „Heutzutage haben die Patienten damit zu kämpfen, dass es viele Informationen gibt“, erklärte Dr. Martin Danner, Bundesgeschäftsführer der BAG Selbsthilfe.
Hier fehle ihnen oft die Orientierung. Ärzte sollten laut Dr. Markus Müschenich, Pädiater und Vorstandsmitglied des Bundesverbands Internetmedizin, die digitalen Neuerungen vor allem als Chance wahrnehmen. „Meine Hoffnung ist die, dass Patienten, die die Ärzte wirklich brauchen, mehr Zeit bekommen", sagte er. Patienten mit banalen Leiden könnten hingegen über die digitale Welt versorgt werden.

\section{Das Ende der Sektorengrenzen}

Die Ärzte haben seiner Meinung nach auch gar nicht die Möglichkeit, sich der neuen Technik zu entziehen. Die Patienten würden sich für beides entscheiden: den klassischen Arztkontakt und ergänzend dazu die mobilen Lösungen, die ihnen mehr Flexibilität und gefühlt Wertschätzung geben. Wenn man als Patient in der Praxis anrufe, erst einmal in der Warteschleife lande, um dann zu erfahren, dass es erst Wochen später einen Termin gebe, fühle man sich „nicht ernst genommen“. Müschenich: „Das ist kein Vorwurf an die Ärzte.“ Die Probleme lägen eher im Gesundheitssystem. Den- noch: „Als digitales Start-up wissen Sie, dass Sie die Patienten begeistern müssen, um am Markt zu bestehen."

„Es ist nicht einfach nur eine neue Technik, es ist ein Kulturwandel, der sich hier vollzieht", warf Birgit Fischer, Hauptgeschäftsführerin beim Verband der forschenden Pharmaunternehmen (vfa) ein. Es erfolge eine Emanzipation der Patienten, die nach Wissen suchten und ihre Krankheiten aktiv managen wollten. Der Vorteil der digitalen Welt sei dabei, dass sie orts- und zeitunabhängig ist. „Damit reißt sie aber auch die Grenzen zwischen den Sektoren ein und stellt das Ende der Arbeitsteilung dar." Hier sieht Fischer die Politik in der Pflicht, Plattformen für Kooperationen, auch mit den Pharmafirmen zu schaffen. Die geplante Telematikinfrastruktur schaffe dies nicht, machte Müschenich klar. „Sie wurde entwickelt, als es noch kein mobile Health gab." Die aktive Kommunikation des Patienten mit dem Arzt komme dort bislang nicht vor. Müschenich: „Es wird eine Plattform geben, die die Gesundheits-Apps bündelt, die Frage ist nur, wer diese betreibt."

RebekkaHöhl

\title{
App-basiert Arbeiten: Mehr Sicherheit in der Versorgung?
}

\section{Eine App-Lösung soll die Sektorengrenzen zwischen Klinik, Praxis und Pflege aufheben. Und gleichzeitig die Dokumentation straffen.}

Die vernetzte Versorgung - von der Klinik bis hin zum Pflegedienst und zurück - ist in Deutschland nach wie vor eher Vision als Realität. Auch, weil die EDV-Systeme der einzelnen Leistungserbringer oft nur mit Mühe den gegenseitigen Datenaustausch ermöglichen. Der ITKonzern IBM will hier nun eine einfache Lösung bieten, die er im amerikanischen Markt bereits getestet hat. Das Unternehmen setzt auf eine Hybrid-Cloud und eine zugehörige App für den einzelnen Arzt oder Pfleger. Die App-Lösung wird an die jeweiligen Arbeitsbereiche angepasst. „Sie gibt einen Überblick über die Abläufe und Aufgaben, die zu erledigen sind“, erklärt Herbert Bunz, IBM Executive Consultant, Mobile Enterprises. In den USA, wo es „Accountable Care Organisations“ gibt, läuft die zugehörige Pflege-App bereits. Es gehören Kliniken,
Ärzte und Pflegedienste dazu, die sich um den kompletten Versorgungsprozess kümmern und Abschläge hinnehmen müssen, wenn Patienten - obwohl verhinderbar - zu schnell wieder in der Klinik landen. Über die Pflege-App werden nicht nur wichtige Aufgaben und Infos direkt an den Mitarbeiter des Pflegedienstes gepuscht, dieser muss auch übers Smartphone in einer Art Checkliste dokumentieren, was er abgearbeitet hat. Zudem können Berichte und Fotos zum Zustand des Patienten hinterlegt werden. Für Nachfragen und Notfälle gibt es die Direktkontaktfunktion zum betreuenden Arzt und Fallmanager. Schließlich hilft die App durch die GPRS-Anbindung und Navi noch dabei, Hausbesuche zeitlich effizienter zu planen.

In der Klinik läuft die Technik ähnlich: Der Arzt bekommt am Krankenbett die Infos, die er benötigt, ohne lange in der Patientenakte suchen zu müssen und kann vor allem schneller Abläufe dokumentieren, da viele Standards schon hinterlegt sind und mit einem Fingertipp abgehakt werden können. Es geht dabei vor allem darum, nichts $\mathrm{zu}$ vergessen. „Die App alleine macht es allerdings nicht“, sagt Bunz deutlich. „Es braucht eine Änderung der Organisation. Man muss Rollen und Prozesse definieren.“ Deshalb macht IBM nur wenige Vorgaben. „Wir entwickeln das System mit Kunden gemeinsam“, so Urs Schollenberger, Business Leader Mobile Enterprise DACH bei IBM.

Die App müsse den Anforderungen der Mitarbeiter gerecht werden, jedoch versuche man, replizierbare Lösungen für einzelne Branchen aufzusetzen. Schollenberger: „Die App ist zu 70 \% vordefiniert, zu $30 \%$ wird sie durch den Kunden gestaltet. “Die Daten für die App werden dabei immer nur fallbezogen und temporär in die zugehörige Cloud gehoben. Dort werden sie konvertiert und an das jeweilige Endgerät des Nutzers übermittelt.

RebekkaHöhl 